\title{
The Spring Lecture: Communicating risks and uncertainty by Sir David John Spiegelhalter
}

\section{Abstract of the lecture}

\author{
[Institute and Faculty of Actuaries, London, May 2016]
}

Good communication is vital, both as an ethical duty and practical contribution to allowing people to make an informed choice. But it is complex, requiring an understanding and respect for a wide range of audiences, and ideally co-producing material with users. I assume that the aim is balanced communication rather than persuasion, although it is naive to assume that education is all that is necessary for people to make decisions that others may consider "rational".

Numbers do not speak for themselves, and the recent story about bacon and cancer illustrates how bad some risk communication can be. The "framing" is vital, and research shows that clear use of absolute risks, preferably expressed as frequencies in a group of similar people, reduces bias and increases understanding. And, if we are serious about balanced communication, the possible benefits and harms must be given equal salience: the recent breast cancer screening leaflets, rather remarkably, exemplify this approach and report both early deaths prevented and over-treatments, and do not explicitly recommend going for screening. All this is expressed as "expected frequencies" in, say, 200 women attending screening, which has been shown to improve reasoning in uncertain environments, and is now in the GCSE maths syllabus.

Another popular metric for risk communication can be called "effective age", or "real age" - the age of an average healthy person with the same risk levels as you. An example is the "UbbLE age", but since this compares you with the mean 5-year mortality risk, which is dominated by people who are already sick, anyone who is healthy comes in at around 10 years less than their chronological age. Since overall hazard increases exponentially at around $9 \%$ per year, a habit with a hazard ratio of 1.09 is effectively equivalent to making you one year older. So lifestyle factors can be easily translated into shifts in effective age.

Not all risks can be well-quantified, and the Intergovernmental Panel on Climate Change, and others, have developed means of qualifying statements with a scale of confidence in an analysis, and I have suggested a rough four-level rating scale. Finally, I illustrate that at times of crisis, it is best not to go in for premature reassurance.

I am very grateful for the support of the Winton Foundation.

The lecture is available to view at: https://www.youtube.com/watch?v=CQrIZyYONOg 